\title{
The Urban Youth Trauma Center: A Trauma-Informed Continuum for Addressing Community Violence Among Youth
}

\author{
Jaleel Abdul-Adil ${ }^{1}$ [D $\cdot$ Liza M. Suárez ${ }^{1}$ \\ Received: 6 May 2020 / Accepted: 9 April 2021 / Published online: 19 April 2021 \\ (c) The Author(s), under exclusive licence to Springer Science+Business Media, LLC, part of Springer Nature 2021
}

\begin{abstract}
Contemporary community violence has escalated into a national public health crisis with urban youth, particularly ethnic minorities, suffering disproportionate negative impacts. The Urban Youth Trauma Center (UYTC) promotes a traumainformed continuum of prevention-to-intervention services that combines community-based and clinic-based manualized protocols designed to reduce and prevent community violence for youth and families. Based on a socio-ecological model, UYTC has the main goals of addressing community violence and related traumatic stress as well as co-occurring conditions of substance abuse and disruptive behavior problems in urban youth by: (1) raising public awareness; (2) disseminating specialized trauma-informed training; and (3) mobilizing service system coalitions. UYTC employs this evidence-based yet flexible structure for disseminating, implementing, and evaluating trauma-informed training as a means of contributing to the reduction and prevention of community violence for low-income urban minority youth and families who bear the biggest burden of this current crisis.
\end{abstract}

Keywords Child trauma $\cdot$ Community violence $\cdot$ Dissemination and implementation $\cdot$ Urban youth $\cdot$ Trauma-informed services

\section{Community Violence Exposure and Adverse Youth Outcomes}

Violence in the United States has currently escalated into a national public health crisis that demands attention across society (Centers for Disease Control and Prevention [CDC], 2017; Freire-Vargas, 2018; Slutkin et al., 2018). Community violence can be defined as "intentional acts of interpersonal violence committed in public areas by individuals who are not intimately related to the victim, including witnessing, learning about or being the victim of shootings in public areas, fights and physical assaults among gangs and other groups, bullying, stabbings, violence in drug deals or robberies, and sexual assault outside of the home" (National Child Traumatic Stress Network [NCTSN], 2014). Community violence is a particularly prevalent type of interpersonal violence in the United States that has recently received

Jaleel Abdul-Adil

adil@uic.edu

1 Department of Psychiatry, Institute for Juvenile Research, University of Illinois at Chicago, 1747 West Roosevelt Road, Mail Code 747, Chicago, IL 60608, USA greater recognition and response among public health agencies in the U.S (Massetti, 2016; Substance Abuse and Mental Health Services Administration [SAMHSA], 2016) due to its association with a wide range of negative outcomes (Finkelhor et al., 2015; Hong et al., 2019; Krug et al., 2002). Multiple indicators suggest contemporary youth face alarming levels of violence, including homicide being one of the top three causes of death for people ages 15-34 years old (CDC, 2019).

Youth exposed to community violence usually experience a myriad of adverse consequences (Buka et al., 2001; Pierre et al., 2020; Voisin et al., 2016) with multiple difficulties reported even among those who are "fortunate" to survive violent exposures and incidents (Jackson et al., 2019; Pierre et al., 2020). Traumatic stress, one of the most commonly reported consequences associated with youth exposed to community violence (Deane et al., 2020; Pahl et al., 2020), is particularly prevalent among urban, low-income ethnic minority youth, many of whom are already suffering from significant socio-economic disparities and poly-victimization vulnerabilities (Andrews et al., 2019; Bernard et al., 2020; Pahl et al., 2020). Community violence remains a disproportionately destructive dynamic in low-income urban 
minority communities, especially among male youth (Gaylord-Harden et al., 2017; Rich, 2009, 2016) with shootings and violence impacting primarily the most disadvantaged minority neighborhoods (Andrews et al., 2019; Pahl et al., 2020) whose poly-victimized youth residents are more likely to meet criteria for psychiatric diagnosis and be involved with court-related delinquency (Charak et al., 2019; Ford \& Delker, 2018). Youth experiencing traumatic reactions and co-occurring problems exhibit greater severity in symptoms, show greater functional impairments, often have unmet treatment needs, and demonstrate greater involvement across multiple service systems (Suarez et al., 2012).

In addition to these ongoing violence-related mental health crises, urban youth exposed to traumatic stressors and community violence are also more likely to suffer disproportionately from the socio-economic upheaval and community unrest during this COVID-19 pandemic. This health emergency has imposed additional adversities for these youth due to strains on already-scarce mental health service structures, aggravations of racial and historical traumas, and exposures to new traumatic incidents including escalating police-minority conflicts (Fortuna et al., 2020; Loeb et al., 2020; Novacek et al., 2020). In light of these compounding youth needs, it is imperative to support prevention and early intervention initiatives that provide trauma informed guidance to youth-serving providers (Collin-Vezina, et al., 2020).

\section{Urban Youth Trauma Center: A Prevention-Intervention Continuum for Community Violence}

Through supports from several funding agencies and various academic-community partnerships, the Urban Youth Trauma Center (UYTC) has been established and developed since 2009 as one resource in the growing national effort to address community violence and related traumatic stress, as well as associated co-occurring conditions in urban youth. Located at the University of Illinois at Chicago (UIC), UYTC was founded through a federal grant from the Substance Abuse and Mental Health Services Administration (SAMHSA) to participate in the National Child Traumatic Stress Network (NCTSN). NCTSN is comprised of a national network of frontline providers, family members, researchers, and national partners who collaboratively develop and deliver evidence-based services for a wide range of traumatic stressors and co-occurring conditions (Pynoos et al., 2008). UYTC has subsequently secured continued support from NCTSN along with targeted city, state, and private funds to develop and expand its structure for dissemination, implementation, and evaluation of provider training and youth programming to provide violence prevention and intervention for urban youth.
The UYTC structure is organized along a prevention-tointervention continuum that integrates protocols for youth needs ranging from primary prevention for at-risk youth in public health settings (e.g., neighborhood schools, afterschool program sites, community agencies, recreational centers, etc.) through escalating difficulty types culminating in intensive intervention for severely disturbed youth in clinical settings (e.g., therapeutic schools, outpatient clinics, inpatient hospitals, residential facilities, etc.). This prevention-intervention continuum of care permits protocols to be designed to "meet youth where they are" to support them with the necessary psychosocial skills to "get them where they need to go." Through training, dissemination, and community engagement activities, UYTC aims to raise awareness about the needs of youth and families exposed to community violence and increase access to trauma informed services in urban communities. The resulting integrated UYTC structure of program development, adaptation, and mobilization of resources is described below.

\section{UYTC Training Model}

UYTC provides rigorous professional training to clinical therapists as well as other youth service providers with a special emphasis on integrated prevention-intervention and trauma-informed continuum of care to address community violence and co-occurring substance abuse and behavioral problems (Dahlberg \& Krug, 2002; Giordano et al., 2016; Regan et al., 2019). UYTC provides training based on a comprehensive socio-ecological model of violence prevention (Dahlberg \& Krug, 2002; David-Ferdon \& Simon, 2014; Mercy et al., 2008; Rosenberg et al., 2006) that recognizes interactions between individuals and their environments involve multiple and reciprocating factors, levels, and pathways of influence that contribute both risk and protective factors for violence related to: (a) the individual (e.g., early exposure to violence, academic problems, impulse control difficulties, etc.); (b) interpersonal relationships (conflict in the home, poor monitoring, exposure to delinquent peers, no access to supportive adults, etc.); (c) the community (unstable housing, neighborhood crime, gang activity, community isolation, etc.); and (d) the society (socioeconomic status, health disparities, exposure to messages that accept and glorify violence, policies that limit access to services, employment or quality educational opportunities, etc.). In addition, UYTC employs a trauma-informed approach for applying these violence prevention strategies based on relational and responsive frameworks that transform the service focus from an antagonistic "what's wrong with you?" to an engaging "what happened to you?" that is often refreshing for troubled youth, and this affirming approach has particular promise when serving stigmatized, injured, and difficult-to-engage 
violence-exposed minority youth (Sonsteng-Person \& Loomis, 2021; Wical et al., 2020). Consequently, UYTC provides trauma-informed, evidence-based best practices for both prevention and intervention that are anchored on this socio-ecological model as a means of integrating multi-level protocols, including national media messages, intensive technical trainings, ongoing post-training consultations, and relevant supplemental resources for serving the continuum of needs of youth and families affected by community violence.

UYTC's training model applies the Theory of Planned Behavior [TPB] (Ajzen \& Schmidt, 2020; Bosnjak et al., 2020; Lindgren et al., 2021) to maximize adoption of new evidence-based practices by provider training participants. UYTC employs TPB to address attitudes, social norms, and perceived controls regarding integrated co-occurring trauma treatments through: (1) presenting research findings to correct mistaken beliefs; (2) including testimonials from significant people and organizations to maximize social approval; and (3) facilitating resources and opportunities to promote perceived personal control (Casper, 2007). To further strengthen community collaborations and partnerships, the UYTC training model also incorporates a "Community of Practice" (CoP) model comprised of collaborative in-person intensive trainings combined with ongoing specialized virtual consultations facilitated by UYTC staff that parallels peer-to-peer public health sector service implementation supports, such as the in-person NCTSN Community-Based Learning Collaborative (Hanson et al., 2016, 2019) and virtual Communities of Practice [CoP] (Barwick et al., 2009; Serhal et al., 2018). Although temporarily modified to allvirtual format during this COVID-19 pandemic, the standard UYTC CoP template remains comprised of in-person beginner/advanced training sessions at project onset followed by both follow-up trainings either online or in-person on regular intervals (monthly, quarterly, semi-annual or yearly) bolstered by regular or ad hoc web-based consultations based on adult learning principles and collaborative educational approaches (Markiewicz et al., 2006).

\section{UYTC Training Goals}

Based on its socioecological model, UYTC adopts two main goals for a trauma-informed and integrated structure that can successfully address the interactive influences of individual, relationship, community, and society factors that contribute to community violence as well as co-occurring problems of disruptive behavior and substance abuse. In particular, UYTC seeks to: (1) raise public awareness about community violence with emphasis (but not limitation) on urban settings (addressing primarily individuals, communities, and society); (2) disseminate specialized trauma-informed training to community-based providers, including paraprofessionals who can supplement services (addressing primarily individuals and relationships). In support of these two goals, UYTC also mobilizes both local and national broad-based service system coalitions for public awareness and training activities that specifically address community violence as well as reduce and prevent general behavioral health disparities among underserved low-income urban ethnic minority youth and their families (addressing primarily communities and society). The remainder of this article will be a brief program description that highlights these two UYTC model components.

\section{Goal \#1: Raise Public Awareness Among Professionals and Consumers}

Although community violence as a general topic has recently received increased public attention, the socio-political complexities of community violence, especially in urban ethnic minority settings, have not been fully integrated into mainstream programming nor general public knowledge and, thus, still require special public attention (Degruy, 2017; Farmer et al., 2006; Lipscomb et al., 2019; Oliver, 2001; Spencer \& Perlow, 2018). The chronic underlying factors of racism, discrimination, poverty, police brutality, and other historic structural inequities continue contributing to community violence among low-income urban minority populations (Aymer, 2016; Calvert et al., 2020; SantiagoRivera et al., 2016), and raising general awareness about the detrimental daily impacts-despite notions of a "postracial society"- -accompanied by suggested progressive responses and strategies that can help reduce or prevent continued systemic problems (Edberg et al., 2017) parallels other public anti-violence messaging involved with "bystander campaigns" and "\#MeToo movement" (Coker et al., 2020). Consequently, trauma-informed program trainings that integrate special emphases on the distinct social, political, and economic factors contributing to community violence and related adversities among racial, ethnic, religious, and other minority groups may maximize program suitability and impact (Aymer, 2016; Comas-Díaz, 2016; Degruy, 2017; Lipscomb \& Ashley, 2018; Rich, 2016). Hence, UYTC strives to raise awareness by promoting public education and prompting collective as well as individual action to reduce and prevent community violence among urban youth and families, particularly minorities, with: (a) public trainings for diverse professional groups (e.g., youth and adult consumers, clinicians, mentors, police, judges, etc.); and (b) coordinated social media campaigns that use popular platforms (Facebook, Instagram, Twitter, etc.) for the general public (including youth, parents, service providers, agency administrators, teachers, public health professionals, politicians, etc.). 


\section{Leadership}

UYTC has adopted both national and local leadership strategies for raising public awareness about community violence prevention and intervention that includes both professional collaboration (Rashid et al., 2009) and public health advocacy (Mizrahi \& Rosenthal, 2001) at local, regional, and national levels (Centers for Disease Control and Prevention, 2019; David-Ferdon et al., 2016). First, regarding professional collaboration leadership, UYTC Co-Directors serve in national leadership positions on professional committees comprising youth service providers (psychologists, psychiatrists, social workers, teachers, nurses, mentors, etc.), parent mental health consumers, agency administrators, legal advocates, and policy officials; and these committees sponsor public events and provider networks designed to share information and resources toward addressing community violence, co-occurring behavioral and substance abuse, and traumatic stress on contemporary youth (e.g., the NCTSN Committees on Community Violence and Substance Abuse). UYTC Co-Directors have also served on city, county, and state professional associations to contribute subject matter expertise as part of collaborative projects to address community violence and related co-occurring conditions among partnering communities. Secondly, regarding public health advocacy leadership, UYTC Co-Directors spearhead the development of NCTSN specialized webinars along with guest appearances on other public access sites (e.g., TV programs, news articles, scholarly conferences, SAMHSA and CDC lectures, etc.) to inform providers, consumers, and policymakers on topics related to community violence and co-occurring conditions. In a recent example, UYTC Co-Directors launched a COVID-19 "pandemic pivot" consisting of disseminating adapted evidence-based practices at both national and local levels through social media campaigns, webinars, trainings, and consultations for supporting urban and youth and families to "survive and thrive" during the pandemic crisis. This "pivot" featured specialized content that adapted core UYTC materials toward addressing these unprecedented healthcare and socioeconomic upheavals that exacerbated existing health disparities, community violence, systemic racism, concentrated poverty, and policeminority conflicts.

\section{Leverage}

In addition to leading professional advocacy and collaboration efforts, UYTC also seeks to leverage existing public awareness activities and social media outlets to amplify visibility and impact of community violence prevention and intervention efforts (Dempsey et al., 2018; Paddock et al., 2017). For example, UYTC annually promotes and participates in the National Youth Violence Prevention Week, an annual public awareness campaign sponsored by Students Against Violence Everywhere (SAVE) as an initiative of the Sandy Hook Promise (Langheim \& McCaughan, 2021). In addition, UYTC maintains an active social media presence (e.g., Facebook, Instagram, Twitter, YouTube, webpages for both UIC and NCTSN, etc.) to facilitate spreading best practice strategies, relevant news articles, and video-based messaging about community violence prevention and intervention as a means of engaging youth, families, providers, and the general public in an appealing contemporary style (Gomez Bravo et al., 2019).

\section{Goal \#2: Disseminate Specialized Trauma-Informed Training for Urban Minority Youth}

In addition to raising public awareness, UYTC provides practical strategies to support effective responses to community violence and co-occurring conditions that negatively impact youth and families, particularly urban minority populations. UYTC has adopted three protocols (e.g., YOUTHCAN, TST-SA and STRONG Families which are described below) that span the continuum of care from prevention to intervention as a means of enabling evidence-based services across the range of needs from urban community-violence exposed populations. Moreover, UYTC has developed an inclusive training protocol permits engaging participants, teaching techniques, and supporting role-based applications across the full spectrum of youth providers (e.g., licensed therapists, community-based paraprofessionals, parents/ guardians with minimal formal behavioral health training, etc.) in settings (homes, clinics, schools, courts, hospitals, faith-based centers, etc.) that can all make valuable contributions toward the cumulative reduction and prevention of community violence and associated adversities among youth and families. Consequently, UYTC promotes the previously described prevention-to-intervention continuum of care perspective to maximize access and effectiveness of services for multiple needs of low-income underserved minority populations. The aforementioned UYTC "COVID-19 pandemic pivot" campaign, which enriched existing UYTC best practices by incorporating COVID-19 healthcare information and telehealth strategies along with social justice and systemic racism responses for community unrest, is a recent example of inclusive training materials that were designed for, then distributed across, multiple sectors of both the youth service system and local community infrastructure.

\section{Prevention}

For its prevention component, UYTC developed a protocol called YOUTH-CAN (Youth Overcoming Urban Trauma and Healing: A Community Action Network). YOUTH-CAN is a violence prevention protocol derived from the World Health 
Organization/Centers for Disease Control and Prevention violence prevention framework (Centers for Disease Control and Prevention, 2019; David-Ferdon et al., 2016) that UYTC Co-Directors and staff systematically adapted into a community-based, trauma-informed, and culturally-competent protocol for specifically addressing community violence among urban youth and families, particularly underserved ethnic minorities. YOUTH-CAN promotes increasing a local community's joint ability to reduce and prevent community violence by: (1) raising awareness about the causes, links, and effects of trauma and community violence; (2) promoting the use of evidence-based best practices for violence prevention and trauma-informed care; (3) promoting participant-level activation and responsiveness to address community violence; and (4) facilitating network, agency, and peer-to-peer collaborations to magnify, reciprocate, and expand individual efforts. YOUTH-CAN brings youth service system representatives (e.g. clinicians, case workers, school personnel, politicians, court judges, probation officers, law enforcement personnel, youth, parents, youth advocates, prosocial activists, mentors, and faith-based groups) into a cooperative UYTC-sponsored structure for collaboratively providing effective trauma-informed and violenceresponsive care for low-income urban ethnic minority youth.

Based on its socioecological model for violence prevention (Dahlberg \& Krug, 2002), YOUTH-CAN addresses the individual, relationship (e.g., peer and family), community, and societal levels of violence prevention by providing a comprehensive framework for applying trauma-informed, evidence-based "Best Practices.". The core best practices consist of: (1) identifying and supporting youth affected by trauma and community violence; (2) developing safe, stable, and nurturing relationships between youth and their parents/ caregivers; (3) developing prosocial life skills and recreational pursuits in youth; (4) promoting safe environments and activities in the local community; and (5) changing cultural and social norms that appear to "promote" or "glorify" violence. UYTC's training, consultation and advanced support for implementing these practices are rooted in the establishment of an Individual Action Plan (IAP) anchored around these Best Practices that sync with other YOUTHCAN participants, agency colleagues, and local community members in support of community violence prevention activation (Dinizulu et al., 2019).

\section{Intervention}

For its intervention component, UYTC developed two protocols called respectively STRONG (Strengths for Trauma Resilience-Overcoming N' Growing) Families and Trauma Systems Therapy for Adolescent Substance Abuse, Suarez (TST-SA). On the externalizing disorders end of the clinical diagnosis continuum, STRONG Families is a manualized clinic-based adaptation of the evidence-based SAFE Children program listed in SAMHSA's National Registry of Evidence-Based Programs and Practices (2009) for addressing traumatic stress, community violence, and co-occurring disruptive behavior disorders including gang violence (AbdulAdil \& Suarez, 2018). On the other internalizing disorders end of the diagnostic continuum, TST-SA is an adaptation of the evidence-based trauma treatment program Trauma Systems Therapy (Saxe et al., 2015) for addressing traumatic stress, community violence, and co-occurring substance abuse (Suarez et al., 2006, 2014). Taken together, these two treatment protocols enable UYTC to offer a complementary continuum of care approach to addressing traumatic stress associated with community violence and related cooccurring conditions ranging from externalizing (STRONG Families) to internalizing (TST-SA) symptomatology.

\section{Innovation}

As part of its applied science structure, UYTC emphasizes flexible fidelity to maintain the integrity of the original evidence-based protocols while collaborating with community partners to make innovative and structured content adaptations that meet precise needs of local populations and providers (Cho et al., 2019; Chorpita, 2019; Chorpita et al., 2005; Fonagy \& Luyten, 2019; Kazdin, 2019; Kendall \& Beidas, 2007; Shelton \& Lee, 2019; Wiltsey Stirman et al., 2017). For example, many urban minority youth and families have been directly or indirectly impacted to some degree by the George Floyd tragedy and related community unrest, and UYTC infused the standard protocol content with enhanced emphases on ethnic identity and resiliency, systemic racism, police brutality, historic racial trauma, traumatic grief, secondary traumatic stress, and social justice mobilization in response to partner communities' needs and requests. Additional recent beneficial innovations from these collaborative and culturally-responsive UYTC frameworks for flexible fidelity include: (1) YOUTH-CAN adaptations in partnership with the Park Forest (IL) Police Department that developed into city-wide trauma training collaborations which include local government agencies, schools, social services, and other key community stakeholders; (2) adaptations of both YOUTH-CAN and STRONG Families protocols based on UYTC staff expertise with popular youth media culture as well as development of an innovative new UYTC protocol named Hip-Hop H.E.A.L.S. (Helping Everyone Achieve Liberation and Success) that delivers standard UYTC protocol content through strategically-selected Rap music and Hip-Hop culture materials designed for reaching and motivating this popular musical genre's youth enthusiasts; and (3) adaptations of selected materials from YOUTH-CAN, STRONG Families, and NCTSN-related trauma-informed products in partnership with Islamic mental health agencies, 
charity institutions, and service providers that guide targeted assessments and specialized services for both indigenous and immigrant Muslim youth and families, including war refugees and other traumatized religious minority populations in both the U.S. and international cities (e.g., Syria, Turkey, Central Asian republics, etc.).

\section{Service System Coalitions: Activation Toward Mobilized and Coordinated Care}

Toward its goals of raising awareness and training providers, UYTC promotes both individual and agency activation through service system coalitions that seek to mobilize and coordinate interagency, multi-disciplinary, and communitywide synergy as well as inform agency and systems policies toward community violence prevention and intervention (Centers for Disease Control and Prevention, 2019; DavidFerdon et al., 2016; Massetti, 2016; Mitchell \& Ryder, 2020; Rosenblatt et al., 2019; Worrall \& Kjaerulf, 2019). In addition to its Co-Directors national and local committee leadership, UYTC established an Advisory Board (McNeish et al., 2019) consisting of representatives from community and consumer groups as well as relevant public health sectors (e.g., mental health, child welfare, juvenile justice, law enforcement, school, primary care, faith-based, youth advocates, mentors, city and state government liaisons, etc.) to facilitate networking, partnering, and promoting traumainformed policy and practice recommendations across the youth service system. UYTC has recently built on the Advisory Board foundation to form the Community Violence and Behavioral Health Disparities Prevention Task Force 2020 that functioned as a system-wide mechanism for exchanging resources and strategies to address entrenched healthcare inequities impacting local partner communities.

\section{Preliminary Evaluation Process}

UYTC activation efforts appear to suggest a promising beginning in disseminating, directing, and highlighting evidence-based practices among youth service providers toward trauma and violence reduction and prevention. For example, UYTC has successfully reached and trained over 24,600 youth service providers in partnership with almost 650 agencies across the country since project inception through a combination of extensive networking, tailored provider and agency engagement, and diverse accessible training formats. In addition, the UYTC Individual Action Plan format for specific concrete application of UYTC evidence-based prevention protocols appear to offer a promising framework for implementing and refining best practice strategies after initial training completion using a well-defined framework for measuring increased training participant activation towards community violence prevention efforts (Dinizulu et al., 2019). Finally, UYTC maintains an active social media presence for highlighting trauma-informed best practices for prevention of violence and co-occurring disorders as reflected in the most recently completed annual program report (2020) that tracked: (a) 182 Facebook posts resulting in 8,411 "likes," an average total reach of 32,990 people, and 1071 "followers"; (b) 45 Twitter posts resulting in nearly 13,000 tweet impressions; and (c) YouTube training videos for asynchronous UYTC best practices explanations resulting in 2320 views.

\section{Conclusion}

UYTC offers an evidence-based yet flexible structure for disseminating, implementing, and evaluating trauma-informed training for youth providers to effectively respond to community violence impacts and co-occurring conditions of disruptive behavior and substance abuse. Based on a socio-ecological model and guided by a continuum of care framework, UYTC promotes an integrated set of evidence-based protocols, strategies, and resources ranging from communitybased prevention to clinic-based intervention. UYTC also combines individual skill development with collective systemic change through collaborative inter-agency networks and advisory board roles that augment training partnerships and service system synergy. Promising preliminary feedback from participating providers and communities suggest that the UYTC trauma-informed structure and its protocols may ultimately make a notable contribution to the reduction and prevention of community violence as a welcome relief to this national public health challenge, including the despair of low-income urban minority youth and families who bear the biggest burden of this current crisis in this COVID-19 climate.

Funding This project was funded by The Substance Abuse and Mental Health Services Administration (SAMHSA Grant \# 1U79SM059297-01).

\section{Declarations}

Conflict of interest The authors declare that they have no known conflict of interest to disclose.

\section{References}

Abdul-Adil, J., \& Suarez, L. M. (2018). Navigating the vortex of community violence: Applying the STRONG families treatment approach for gang-involved youth. In J. D. Osofsky, \& B. M. Groves (Eds.), Violence and trauma in the lives of children: Overview of exposure (pp. 99-119). Praeger/ABC-CLIO. 
Ajzen, I., \& Schmidt, P. (2020). Changing behaviour using the theory of planned behavior (pp. 17-31). Cambridge University Press.

Andrews, A. R., Lopez, C. M., Snyder, A., Saunders, B., \& Kilpatrick, D. G. (2019). Polyvictimization, related symptoms, and familial and neighborhood contexts as longitudinal mediators of racial/ethnic disparities in violence exposure across adolescence. Journal of Immigrant and Minority Health, 21(4), 679-692.

Aymer, S. R. (2016). "I can't breathe": A case study-Helping black men cope with race-related trauma stemming from police killing and brutality. Journal of Human Behavior in the Social Environment, 26(3-4), 367-376.

Barwick, M. A., Peters, J., \& Boydell, K. (2009). Getting to uptake: Do communities of practice support the implementation of evidencebased practice? Journal of the Canadian Academy of Child and Adolescent Psychiatry, 18(1), 16.

Bernard, D. L., Calhoun, C. D., Banks, D. E., Halliday, C. A., HughesHalbert, C., \& Danielson, C. K. (2020). Making the "c-ACE" for a culturally-informed adverse childhood experiences framework to understand the pervasive mental health impact of racism on Black youth. Journal of Child \& Adolescent Trauma. https://doi.org/10. 1007/s40653-020-00319-9.

Bosnjak, M., Ajzen, I., \& Schmidt, P. (2020). The theory of planned behavior: Selected recent advances and applications. Europe's Journal of Psychology, 16(3), 352-356.

Buka, S. L., Stichick, T. L., Birdthistle, I., \& Earls, F. J. (2001). Youth exposure to violence: Prevalence, risks, and consequences. American Journal of Orthopsychiatry, 71(3), 298-310.

Calvert, C. M., Brady, S. S., \& Jones-Webb, R. (2020). Perceptions of violent encounters between police and young Black men across stakeholder groups. Journal of Urban Health, 97, 279-295.

Casper, E. S. (2007). The theory of planned behavior applied to continuing education for mental health professionals. Psychiatric Services, 58(10), 1324-1329.

Centers for Disease Control and Prevention. (2017). Violence prevention-National Violent Death Reporting System. https://www.cdc. gov/violenceprevention/nvdrs/index.html. Updated 2019.

Centers for Disease Control and Prevention. (2019). National center for injury prevention and control. Preventing youth violence. https://www.cdc.gov/violenceprevention/pdf/yv-factsheet508. pdf. Updated 2019.

Charak, R., Ford, J. D., Modrowski, C. A., \& Kerig, P. K. (2019). Polyvictimization, emotion dysregulation, symptoms of posttraumatic stress disorder, and behavioral health problems among justiceinvolved youth: A latent class analysis. Journal of Abnormal Child Psychology, 47(2), 287-298.

Chorpita, B. F. (2019). Commentary: Metaknowledge is power: Envisioning models to address unmet mental health needs: Reflections on Kazdin (2019). Journal of Child Psychology and Psychiatry, 60(4), 473-476.

Chorpita, B. F., Daleiden, E. L., \& Weisz, J. R. (2005). Identifying and selecting the common elements of evidence-based interventions: A distillation and matching model. Mental Health Services Research, 7(1), 5-20.

Cho, E., Wood, P. K., Taylor, E. K., Hausman, E. M., Andrews, J. H., \& Hawley, K. M. (2019). Evidence-based treatment strategies in youth mental health services: Results from a national survey of providers. Administration and Policy in Mental Health and Mental Health Services Research, 46(1), 71-81.

Coker, A. L., Bush, H. M., Brancato, C. J., Huang, Z., Clear, E. R., \& Follingstad, D. R. (2020). Longer term impact of bystander training to reduce violence acceptance and sexism. Journal of School Violence, 19(4), 525-538.

Collin-Vézina, D., Brend, D., Black, K., Beeman, I., \& Brown, S. (2020). Impacts of child welfare worker and clientele characteristics on attitudes toward trauma informed-care. Developmental Child Welfare. https://doi.org/10.1177/2516103220963139
Comas-Díaz, L. (2016). Racial trauma recovery: A race-informed therapeutic approach to racial wounds. In A. N. Alvarez, C. T. H. Liang, \& H. A. Neville (Eds.), Cultural, racial, and ethnic psychology book series. The cost of racism for people of color: Contextualizing experiences of discrimination (pp. 249-272). American Psychological Association.

Dahlberg, L. L., \& Krug, E. G. (2002). Violence-A global public health problem. In E. Kurg, L. L. Dahlberg, J. A. Mercy, A. B. Zwi, \& R. Lorano (Eds.), World Report on Violence and Health (pp. 1-56). World Health Organization.

David-Ferdon, C., \& Simon, T. R. (2014). Preventing youth violence: Opportunities for action. National Center for Injury Prevention and Control.

David-Ferdon, C., Vivole-Kantor, A. M., Dahlberg, L. L., Marshall, K. J., Rainford, N., \& Hall, J. E. (2016). A comprehensive technical package for the prevention of youth violence and associated risk behaviors. https://www.cdc.gov/violenceprevention/pdf/yv-techn icalpackage.pdf

Deane, K. C., Richards, M., Bocanegra, K., Santiago, C. D., Scott, D., Zakaryan, A., \& Romero, E. (2020). Mexican American urban youth perspectives on neighborhood stressors, psychosocial difficulties, and coping: En sus propias palabras. Journal of Child and Family Studies, 29, 1780-2179.

Degruy, J. (2017). Post-traumatic Slave Syndrome: America's legacy of enduring injury. Caban Productions.

Dempsey, R. C., McAlaney, J., \& Bewick, B. M. (2018). A critical appraisal of the social norms approach as an interventional strategy for health-related behavior and attitude change. Frontiers in Psychology, 9, 2180.

Dinizulu, S. M., Suarez, L. M., Simpson, D., Abdul-Adil, J., \& Jacobson, K. C. (2019). Psychometric properties of the Community Violence-Prevention Activation Measure (CV-PAM): Evaluating provider activation toward community violence prevention. Journal of Community Psychology, 48(2), 545-561.

Edberg, M. C., Cleary, S. D., Andrade, E. L., Evans, W. D., Simmons, L. K., \& Cubilla-Batista, I. (2017). Applying ecological positive youth development theory to address co-occurring health disparities among immigrant Latino youth. Health Promotion Practice, 18(4), 488-496.

Farmer, P. E., Nizeye, B., Stulac, S., \& Keshavjee, S. (2006). Structural violence and clinical medicine. PLoS Medicine, 3(10), e449.

Finkelhor, D., Turner, H. A., Shattuck, A., \& Hamby, S. L. (2015). Prevalence of childhood exposure to violence, crime, and abuse: Results from the national survey of children's exposure to violence. JAMA Pediatrics, 169, 746-754.

Fonagy, P., \& Luyten, P. (2019). Fidelity vs. flexibility in the implementation of psychotherapies: Time to move on. World Psychiatry, 18(3), 270.

Ford, J. D., \& Delker, B. C. (2018). Polyvictimization in childhood and its adverse impacts across the lifespan: Introduction to the special issue. Journal of Trauma \& Dissociation, 19(3), 275-288.

Fortuna, L. R., Tolou-Shams, M., Robles-Ramamurthy, B., \& Porche, M. V. (2020). Inequity and the disproportionate impact of COVID-19 on communities of color in the United States: The need for a trauma-informed social justice response. Psychological Trauma: Theory, Research, Practice, and Policy, 12(5), 443-445.

Freire-Vargas, L. (2018). Violence as a public health crisis. AMA Journal of Ethics, 20(1), 25-28.

Gaylord-Harden, N. K., So, S., Bai, G. J., Henry, D. B., \& Tolan, P. H. (2017). Examining the pathologic adaptation model of community violence exposure in male adolescents of color. Journal of Clinical Child \& Adolescent Psychology, 46(1), 125-135.

Giordano, A. L., Prosek, E. A., Stamman, J., Callahan, M. M., Loseu, S., Bevly, C. M., Cross, K., Woehler, E. S., Calzada, R. M., \& Chadwell, K. (2016). Addressing trauma in substance abuse 
treatment. Journal of Alcohol and Drug Education, 60(2), $55-71$.

Gomez Bravo, R., Gomez Bravo, M., Lygidakis, C., \& Vögele, C. (2019). Social media as an opportunity for public health interventions: The\# Metoo movement as an exemplar. Journal of the International Society for Telemedicine and EHealth, 7(e5), 1-7.

Hanson, R. F., Schoenwald, S., Saunders, B. E., Chapman, J., Palinkas, L. A., Moreland, A. D., \& Dopp, A. (2016). Testing the Community-Based Learning Collaborative (CBLC) implementation model: A study protocol. International Journal of Mental Health Systems, 10(1), 52.

Hanson, R. F., Saunders, B. E., Ralston, E., Moreland, A. D., Peer, S. O., \& Fitzgerald, M. M. (2019). Statewide implementation of child trauma-focused practices using the community-based learning collaborative model. Psychological Services, 16(1), 170.

Hong, J. S., Lee, J. J., Kim, J., Iadipaolo, A. S., Espelage, D. L., \& Voisin, D. R. (2019). Posttraumatic stress, academic performance, and future orientation as pathways to community violence exposure and sexual risk among African American youth in Chicago's Southside. Behavioral Medicine. https://doi.org/10.1080/08964 289.2019.1601610

Jackson, D. B., Posick, C., \& Vaughn, M. G. (2019). New evidence of the nexus between neighborhood violence, perceptions of danger, and child health. Health Affairs, 38(5), 746-754.

Kazdin, A. (2019). Expanding mental health services through novel models of intervention delivery. Journal of Child Psychology and Psychiatry, 60, 455-472.

Kendall, P. C., \& Beidas, R. S. (2007). Smoothing the trail for dissemination of evidence-based practices for youth: Flexibility within fidelity. Professional Psychology: Research and Practice, $38,13-20$.

Krug, E. G., Mercy, J. A., Dahlberg, L. L., \& Zwi, A. B. (2002). The world report on violence and health. The Lancet, 360(9339), 1083-1088.

Langheim, M. R., \& McCaughan, A. M. (2021). Tightening the gap: Advocating for law enforcement and school personnel for identifying students at risk of violence. In Research anthology on school shootings, peer victimization, and solutions for building safer educational institutions (pp. 257-279). IGI Global.

Lindgren, K. P., DiBello, A. M., Peterson, K. P., \& Neighbors, C. (2021). Theory-driven interventions: How social cognition can help. In The handbook of alcohol use (pp. 485-510). Elsevier.

Lipscomb, A. E., \& Ashley, W. (2018). Black male grief through the lens of racialization and oppression: Effective instruction for graduate clinical programs. International Research in Higher Education, 3(2), 51-60.

Lipscomb, A. E., Emeka, M., Bracy, I., Stevenson, V., Lira, A., Gomez, Y. B., \& Riggins, J. (2019). Black male hunting! A phenomenological study exploring the secondary impact of police induced trauma on the Black man's psyche in the United States. Journal of Sociology, 7(1), 11-18.

Loeb, T. B., Ebor, M. T., Smith, A. M., Chin, D., Novacek, D. M., Hampton-Anderson, J. N., Norwood-Scott, E., Hamilton, A.B., Brown, A. F., \& Wyatt, G. E. (2020). How mental health professionals can address disparities in the context of the COVID-19 pandemic. Traumatology. https://doi.org/10.1037/trm0000292.

Markiewicz, J., Ebert, L., Ling, D., Amaya-Jackson, L., \& Kisiel, C. (2006). Learning collaborative toolkit. National Center for Child Traumatic Stress.

Massetti, G. M. (2016). Preventing violence among high-risk youth and communities with economic, policy, and structural strategies. MMWR Supplements. https://doi.org/10.15585/mmwr. su6501a9

McNeish, R., Rigg, K. K., Tran, Q., \& Hodges, S. (2019). Community-based behavioral health interventions: Developing strong community partnerships. Evaluation and Program Planning, 73, 111-115.

Mercy, J. A., Butchart, A., Rosenberg, M. L., Dahlberg, L., \& Harvey, A. (2008). Preventing violence in developing countries: A framework for action. International Journal of Injury Control and Safety Promotion, 15(4), 197-208.

Mitchell, R. J., \& Ryder, T. (2020). Rethinking the public health model for injury prevention. Injury Prevention, 26(1), 2-4.

Mizrahi, T., \& Rosenthal, B. B. (2001). Complexities of coalition building: Leaders' successes, strategies, struggles, and solutions. Social Work, 46(1), 63-78.

National Child Traumatic Stress Network. (2014). Community violence: Reactions and actions in dangerous times. National Child Traumatic Stress Network. Retrieved from http://www.nctsn.org/ sites/default/files/assets/pdfs/community_violence_reactions_ actions.pdf

Novacek, D. M., Hampton-Anderson, J. N., Ebor, M. T., Loeb, T. B., \& Wyatt, G. E. (2020). Mental health ramifications of the COVID-19 pandemic for Black Americans: Clinical and research recommendations. Psychological Trauma: Theory, Research, Practice, and Policy, 12(5), 449-451.

Oliver, W. (2001). Cultural racism and structural violence: Implications for African Americans. Journal of Human Behavior in the Social Environment, 4(2-3), 1-26.

Paddock, E., Samuels, J., Vinik, N., \& Overton, S. (2017). Federal actions to engage communities in reducing gun violence. Joint Center for Political and Economic Studies. Retrieved from https:// www.urban.org/sites/default/files/publication/98519/federal_actio ns_to_engage_communities-in-reducing-gun-violence_1.pdf

Pahl, K., Williams, S. Z., Lee, J. Y., Joseph, A., \& Blau, C. (2020). Trajectories of violent victimization predicting PTSD and comorbidities among urban ethnic/racial minorities. Journal of Consulting and Clinical Psychology, 88(1), 39-47.

Pierre, C. L., Burnside, A., \& Gaylord-Harden, N. K. (2020). A longitudinal examination of community violence exposure, school belongingness, and mental health among African-American adolescent males. School Mental Health, 12, 388-399.

Pynoos, R. S., Fairbank, J. A., Steinberg, A. M., Amaya-Jackson, L., Gerrity, E., Mount, M. L., \& Maze, J. (2008). The National Child Traumatic Stress Network: Collaborating to improve the standard of care. Professional Psychology: Research and Practice, 39(4), 389.

Rashid, J. R., Spengler, R. F., Wagner, R. M., Melanson, C., Skillen, E. L., Mays, R. A., Jr., Heurtin-Roberts, S., \& Long, J. A. (2009). Eliminating health disparities through transdisciplinary research, cross-agency collaboration, and public participation. American Journal of Public Health, 99(11), 1955-1961.

Regan, J., Park, A. L., \& Chorpita, B. F. (2019). Choices in treatment integrity: Considering the protocol and consultant recommendations in child and adolescent therapy. Journal of Clinical Child \& Adolescent Psychology, 48(sup1), S79-S89.

Rich, J. A. (2009). Wrong place, wrong time: Trauma and violence in the lives of young Black men. JHU Press.

Rich, J. A. (2016). Moving toward healing: Trauma and violence and boys and young men of color. Drexel University School of Public Health.

Rosenberg, M. L., Butchart, A., Mercy, J., Narasimhan, V., Waters, H., Marshall, M. S., Jamison, D. T., Breman, J. G., \& Measham, A. R. (2006). Interpersonal violence. Disease Control Priorities in Developing Countries, 2, 755-770.

Rosenblatt, M. S., Joseph, K. T., Dechert, T., Duncan, T. K., D’Andrea, K. J., Stewart, R. M., \& Cooper, Z. R. (2019). American Association for the Surgery of Trauma Prevention Committee topical update: Impact of community violence exposure, intimate partner violence, hospital-based violence intervention, building 
community coalitions and injury prevention program evaluation. Journal of Trauma and Acute Care Surgery, 87(2), 456-462.

Santiago-Rivera, A. L., Adames, H. Y., Chavez-Dueñas, N. Y., \& Benson-Flórez, G. (2016). The impact of racism on communities of color: Historical contexts and contemporary issues. In A. N. Alvarez, C. T. H. Liang, \& H. A. Neville (Eds.), Cultural, racial, and ethnic psychology book series. The cost of racism for people of color: Contextualizing experiences of discrimination (pp. 229-245). American Psychological Association.

Saxe, G. N., Ellis, B. H., \& Brown, A. D. (2015). Trauma systems therapy for children and teens. Guilford Publications.

Serhal, E., Arena, A., Sockalingam, S., Mohri, L., \& Crawford, A. (2018). Adapting the consolidated framework for implementation research to create organizational readiness and implementation tools for project ECHO. The Journal of Continuing Education in the Health Professions, 38(2), 145.

Shelton, R. C., \& Lee, M. (2019). Sustaining evidence-based interventions and policies: Recent innovations and future directions in implementation science. American Journal of Public Health. https://doi.org/10.2105/AJPH.2018.304913

Slutkin, G., Ransford, C., \& Zvetina, D. (2018). How the health sector can reduce violence by treating it as a contagion. AMA Journal of Ethics, 20(1), 47-55.

Sonsteng-Person, M., \& Loomis, A. M. (2021). The role of traumainformed training in helping Los Angeles teachers manage the effects of student exposure to violence and trauma. Journal of Child \& Adolescent Trauma. https://doi.org/10.1007/ s40653-021-00340-6

Spencer, Z., \& Perlow, O. N. (2018). Reconceptualizing historic and contemporary violence against African-Americans as savage white American terror (SWAT). Journal of African American Studies, 22(2-3), 155-173.

Suarez, L. M., Belcher, H. M., Briggs, E. C., \& Titus, J. C. (2012). Supporting the need for an integrated system of care for youth with co-occurring traumatic stress and substance abuse problems. American Journal of Community Psychology, 49(3-4), 430-440.

Suárez, L. M., Saxe, G., Ehrenreich, J., \& Barlow, D. (2006). Trauma systems therapy for adolescent substance abuse. Center for Anxiety and Related Disorders and Boston University.
Suárez, L. M., Ellis, B. H., \& Saxe, G. N. (2014). Integrated treatment of traumatic stress and substance abuse problems among adolescents. In J. Ehrenreich-May, \& B. C. Chu (Eds.), Transdiagnostic treatments for children and adolescents: Principles and practice (pp. 339-362). The Guilford Press.

Substance Abuse and Mental Health Services Administration. (2009). National registry of evidence-based programs and practices. Downloaded August 1, 2009, from http://nrepp.samhsa.gov

Substance Abuse and Mental Health Services Administration. (2016). Tips for survivors: Coping with grief after community violence. Retrieved from https://store.samhsa.gov/product/Coping-WithGrief-After-Community-Violence/SMA14-4888

Voisin, D. R., Patel, S., Hong, J. S., Takahashi, L., \& Gaylord-Harden, N. (2016). Behavioral health correlates of exposure to community violence among African-American adolescents in Chicago. Children and Youth Services Review, 69, 97-105.

Wical, W., Richardson, J., \& Bullock, C. (2020). A credible messenger: The role of the violence intervention specialist in the lives of young black male survivors of violence. Violence and Gender, $7(2), 66-69$.

Wiltsey Stirman, S., Gamarra, J. M., Bartlett, B. A., Calloway, A., \& Gutner, C. A. (2017). Empirical examinations of modifications and adaptations to evidence-based psychotherapies: Methodologies, impact, and future directions. Clinical Psychology: Science and Practice, 24(4), 396-420.

Worrall, R., \& Kjaerulf, F. (2019). Transforming minds, people, and places: Leadership coalition building as catalyst for intersectoral collaboratives in urban violence prevention. Aggression and Violent Behavior, 47, 282-292.

Publisher's Note Springer Nature remains neutral with regard to jurisdictional claims in published maps and institutional affiliations. 